\title{
ARTICLE
}

Cite this: DOI: $10.1039 / \mathrm{x} 0 \times x \times 0000 x$

\section{Reversible Vapochromic Response of Polymer Films Doped with a Highly-Emissive Molecular Rotor}

\author{
Pierpaolo Minei, ${ }^{\mathrm{a}}$ Matthias Koenig, ${ }^{\mathrm{b}}$ Antonella Battisti, ${ }^{\mathrm{c}}$ Muzaffer Ahmad, ${ }^{\mathrm{a}}$ Vin- \\ cenzo Barone, ${ }^{\mathrm{a}}$ Tomas Torres ${ }^{\mathrm{d}, \mathrm{e}}$ Dirk M. Guldi, ${ }^{\mathrm{b}}$ Giuseppe Brancato, ${ }^{\mathrm{a}}$ Giovanni \\ Bottari, ${ }^{\mathrm{d}, \mathrm{e}, *}$ Andrea Pucci ${ }^{\mathrm{f}, \mathrm{g}, *}$
}

Received 00th January 2014

Accepted 00th January 2014

DOI: $10.1039 / \mathrm{x} 0 \mathrm{xx} 00000 \mathrm{x}$

We report on a new vapochromic system suitable for sensing volatile organic compounds (VOCs) based on polymer films doped with 4-(diphenylamino)phthalonitrile (DPAP), a fluorescent molecular rotor sensitive to both solvent polarity and viscosity. Poly(methyl methacrylate) (PMMA) and polycarbonate (PC) films containing small amounts of DPAP $(\leq 0.1 \mathrm{wt} . \%)$ were prepared and exposed to saturated atmospheres of different VOCs. DPAP/PMMA films show a good and reversible vapochromism when exposed to VOCs with high polarity index and favourable interaction with the polymer matrix such as $\mathrm{THF}, \mathrm{CHCl}_{3}$, and acetonitrile. Analogously, DPAP/PC films exposed to polar and highly polymer-interacting solvents, that is, toluene, THF, and $\mathrm{CHCl}_{3}$ show a gradual decrease and red-shift of the emission. Contrary to DPAP/PMMA films, an unexpected increase and further red-shift of fluorescence is observed at longer exposure times as a consequence of an irreversible, solvent-induced crystallization process of PC. The vapochromism of DPAP-doped films is rationalized on the basis of alterations of the rotor intramolecular motion and polarity effects stemming from the environment, which, in concert, influence the deactivation pathways of the DPAP intramolecular charge transfer state. Overall, the present results support the use of DPAP-enriched plastic films as a new chromogenic material suitable for the detection of VOCs.

\section{Introduction}

Nowadays, the detection of volatile organic compounds (VOCs) is an important aspect considering that VOCs are continuously released to the environment by different sources like industrial processes, transportation, agriculture, etc. ${ }^{1,2}$ Some of them have adverse effects on human health. ${ }^{3,4}$ Various detection methods have been proposed in recent years. ${ }^{5-7}$ Leading examples are based on changes in electrochemical, conducting, and chromic properties of the corresponding sensor matrices. In this context, colorimetric sensor systems are of particular interest thanks to their effectiveness and simplicity. ${ }^{8,9}$ To this end, arrays of metalloporphyrins, chromogenic dyes, polymers, ${ }^{10,11}$ and $\mathrm{pH}$ indicators are utilized to detect several VOCs. However, most of these systems are costly, and have significant limitations in the sensor interaction with the analyte.

New strategies for the colorimetric and specific detection of VOCs, which are based on dyes or conjugated polymers embedded in plastic materials, have been reported. ${ }^{12}$ None of the aforementioned assays enable visual detection of VOCs without, however, using software programs to deconvolute the changes in colour pattern taking place during exposure to VOCs.

Notable is the fact that the optical behaviour of these polymerbased VOCs sensors is tuneable by specific interactions between the polymer matrix and the dye through exciton coupling and selforganization. ${ }^{13-15}$ These synergic interactions of the dye with the polymer structure also provide innovative material features and responsive character upon external stimuli. ${ }^{16-18}$ The success of such dye/polymer systems is largely due to the ability of VOCs to spread rapidly inside the polymer matrix and to interact with the dye providing the means for a fast and reliable response..$^{19-21}$ Moreover, most of the polymers are colourless with good film forming features, which allows preparing large area devices under ambient conditions and by means of low cost fabrication techniques.

A particular interesting class of vapochromic molecules are the fluorescent molecular rotors (FMRs). ${ }^{22-25}$ FMRs are flexible chromophores with a fluorescence response that depends on the local viscosity of the environment. ${ }^{26-28}$ FMRs have become rather popular in the last 5-10 years thanks to their easy applicability as nonmechanical viscosity sensors, tools for protein characterization, and local microviscosity imaging. ${ }^{29-37}$ Remarkably, their sensitivity towards viscosity and viscosity changes has reached a precision comparable to that of commercial mechanical rheometers with shorter measurement time. ${ }^{23}$

$p$ - $N, N$-dimethylaminobenzonitrile (dicyanovinyl)julolidine

(DCVJ),

(DMABN),

$9-$ cyanovinyl)julolidine (CCVJ) and tetraphenylethylene (TPE) are among the most known examples of FMRs. ${ }^{24,30-32,38-42}$ Recently, a prototype for a new class of FMRs, namely 4(diphenylamino)phthalonitrile (DPAP) (Fig. 1a), was reported and its sensitivity towards solvent polarity and viscosity was probed using photophysical and computational methods. ${ }^{43}$

DPAP presents a contrasting deactivation pattern of the intramolecular charge transfer (ICT) state in low or high polar media. On one hand, in low and medium polar solvents DPAP shows a strong emission. On the other hand, in high polar and protic solvents the rotor's ICT state is stabilized and decays primarily non-radiatively. In addition to the aforementioned trends, an increase in DPAP emission was observed upon increasing the solvent viscosity as a result of a decrease of rotor flexibility, which in turn favors a radiative deactivation process. More recently, amorphous aggregationinduced emission (AIE) nanoparticles based on DPAP have been 
prepared and were transformed into highly emissive, rhomboidal nanocrystals using an ultrasound stimulus. ${ }^{44}$

While there are widespread applications of FMRs as viscosity sensors, there are scarce examples of their use in combination with polymer matrices.

Herein, we report on the emission properties of DPAP dispersed (0.05-0.1 wt.\%) within transparent thermoplastic matrices such as poly(methyl methacrylate) (PMMA) and polycarbonate (PC) as a function of the exposure to different VOCs. The results are discussed in terms of sensitivity and reproducibility of the vapochromic response of the polymer systems.

\section{Materials and Methods}

Materials

DPAP was prepared following the synthetic procedures reported in the literature. ${ }^{43}$ All the solvents ( $n$-hexane, toluene, acetonitrile $\left(\mathrm{CH}_{3} \mathrm{CN}\right)$, tetrahydrofuran (THF), chloroform $\left.\left(\mathrm{CHCl}_{3}\right)\right)$ were purchased from Sigma-Aldrich and used as received. Poly(methyl methacrylate) (PMMA, Aldrich, $M w=350,000 \mathrm{~g} / \mathrm{mol}$, acid number $<1 \mathrm{mg} \mathrm{KOH} / \mathrm{g}$ ) and random copolymer polycarbonatepolysiloxane LEXAN ${ }^{\circledR}$ EXL 1414T (PC, SABIC, $M w=220,000$ $\mathrm{g} / \mathrm{mol}$ with $1.5 \mathrm{wt} . \% \mathrm{Si}$ ) were used as received.

The solvents polarity index, the PMMA-solvent Flory-Huggins interaction parameter $\chi$, and the solubility parameter difference $\Delta \delta$ (in the case of PC) are reported in Table 1.

Table 1 Vapour pressure of different solvents at $20{ }^{\circ} \mathrm{C},{ }^{45}$ polarity index, ${ }^{46}$ PMMA-solvent Flory-Huggins interaction parameter $\chi{ }^{47}$ and solubility parameter difference $\Delta \delta$ (for PC) ${ }^{48}$ for utilized solvents

\begin{tabular}{|c|c|c|c|c|}
\hline solvent & $\begin{array}{c}\text { vapour } \\
\text { pressure } \\
(\mathrm{mm} \mathrm{Hg})\end{array}$ & $\begin{array}{c}\text { polarity } \\
\text { index }\end{array}$ & $\chi^{\mathrm{a}}$ & $\begin{array}{c}\Delta \delta \\
\left(\left(\mathrm{cal} / \mathrm{cm}^{3}\right)^{1 / 2}\right)^{\mathrm{b}}\end{array}$ \\
\hline$n$-hexane & 124 & 0.1 & $0.530^{49}$ & 2.5 \\
\hline toluene & 28.5 & 2.4 & 0.450 & 0.9 \\
\hline THF & 142 & 4.0 & 0.494 & 0.7 \\
\hline $\mathrm{CHCl}_{3}$ & 158.4 & 4.1 & 0.44 & 0.5 \\
\hline $\mathrm{CH}_{3} \mathrm{CN}$ & 88.8 & 5.1 & $0.500^{49}$ & -3 \\
\hline
\end{tabular}

${ }^{\mathrm{a}}$ for PMMA; ${ }^{\mathrm{b}}$ for PC; $\Delta \delta=\delta_{\mathrm{PC}}-\delta_{\text {solvent }} ; \delta_{\mathrm{PC}}=9.8\left(\mathrm{cal} / \mathrm{cm}^{3}\right)^{1 / 2}$

\section{Preparation of polymer films ${ }^{16}$}

$500 \mathrm{mg}$ of PMMA were dissolved in $15 \mathrm{~mL}$ of chloroform under stirring for 10 minutes. Then, DPAP ( 0.05 or $0.1 \mathrm{wt} . \%)$ was added and the resulting solution casted into clean Teflon ${ }^{\circledR}$ Petri-dishes. In this way, 90-120 $\mu \mathrm{m}$ thick films of DPAP/PMMA were realized after solvent evaporation. The same procedure was adopted for the preparation of DPAP/PC films, save that dichloromethane was used as solvent for PC and clean glass Petri-dishes for film formation.

\section{Apparatus and Methods}

UV-Vis spectra of DPAP/polymer films were recorded on a Perkin Elmer Lambda 650 at room temperature. Fluorescence spectra $\left(\lambda_{\text {exc }}\right.$ $=325 \mathrm{~nm}$ ) of DPAP/polymer films were measured on a Horiba Jobin-Yvon Fluorolog ${ }^{\mathbb{B}}-3$ spectrofluorometer at room temperature in the dark by using the F-3000 Fibre Optic Mount apparatus coupled with optical fibre bundles. Light generated from the excitation spectrometer is directly focused to the DPAP/polymer sample using an optical fibre bundles. Emission from the sample is then directed back through the bundle into the collection port of the sample compartment.

The emission response of the dye/polymer films was tested by exposing a $2 \times 2 \mathrm{~cm}$ DPAP/polymer film, attached to an aluminium foil covering a $50 \mathrm{~mL}$ closed container (Fig. $1 \mathrm{~b}$ ) ${ }^{50}$, to $20 \mathrm{~mL}$ of five organic solvents of different polarity at ambient temperature (20 $\left.{ }^{\circ} \mathrm{C}\right)$ and atmospheric pressure, namely $n$-hexane, toluene, THF, $\mathrm{CHCl}_{3}$, and $\mathrm{CH}_{3} \mathrm{CN}$, with similar conditions for each experiment. The experiments were collected after solvent saturation was reached. The concentration of about $10^{4} \mathrm{ppm}$ for toluene and $10^{5}$ ppm for the other solvents were estimated taking into consideration their vapour pressures listed in Table 1.

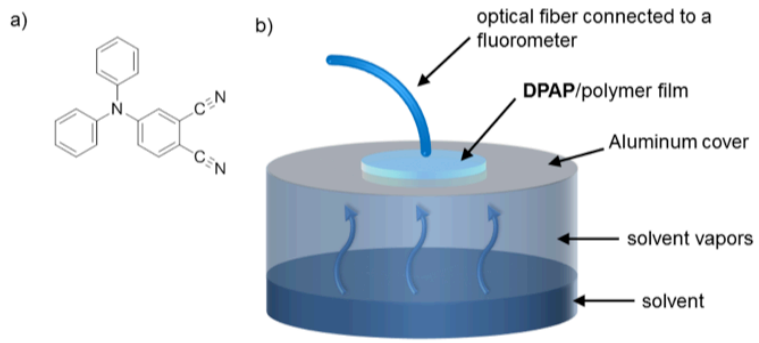

Fig. 1 a) Molecular structure of 4-(diphenylamino)phthalonitrile (DPAP), b) Schematic representation of the apparatus used to study the vapochromic behaviour of the DPAP/polymer films.

Microscopy images and lifetime measurements were collected by using a Leica TCS SP5 SMD inverted confocal microscope (Leica Microsystems AG, Wetzlar, Germany) equipped with an external pulsed diode laser (PicoQuant $\mathrm{GmbH}$, Berlin, Germany) for excitation at $405 \mathrm{~nm}$. The laser repetition rate was set to be $40 \mathrm{MHz}$. Each of the image sizes were $512 \times 512$ pixels and acquired with a scan speed of $400 \mathrm{~Hz}$ (lines per second). The pinhole aperture was set at 1.00 Airy. DPAP/polymer films fixed on microscope glass slides were viewed with a $100 \times 1.3 \mathrm{NA}$ oil immersion objective (Leica Microsystems). The images were collected using low excitation power at the sample $(10-20 \mu \mathrm{W})$. Emissions were monitored in the $430-490 \mathrm{~nm}$ range by acousto-optical tuneable beam splitter (AOBS) based built in detectors. Acquisition lasted until about 100-200 photons per pixel were collected, at photon counting rates of 100-500 kHz. Emission lifetime images (FLIM) of the DPAP/polymer were elaborated using Picoquant Symphotime software for FLIM analysis.

Differential scanning calorimetry (DSC) was carried out under nitrogen atmosphere by using a Mettler DSC 30 instrument. Samples of $10-20 \mathrm{mg}$ were heated from 40 to $300{ }^{\circ} \mathrm{C}$ at $10^{\circ} \mathrm{C} / \mathrm{min}$. Melting enthalpies were evaluated from the integrated areas of melting peaks by using indium for calibration. PC crystalline content $\left(f_{c}\right)$ was evaluated from the measured melting enthalpy $\left(\Delta \mathrm{H}_{\mathrm{m}}\right)$ taking into account the melting enthalpy of the perfect PC crystal $\Delta \mathrm{H}_{\mathrm{m}}^{0}$ $=132 \mathrm{~J} / \mathrm{g},{ }^{51}$ using the equation 1 :

$$
f_{c}=\frac{\Delta H_{m}}{\Delta H_{m}^{0}} \cdot 100
$$

\section{Results and Discussion}

The solvatochromism of DPAP in various solvents has been fully described in a previous study by some of us. ${ }^{43}$ In the following the main results are summarized. DPAP shows a solvent-insensitive absorption with a broad band maximizing at around $325 \mathrm{~nm} .{ }^{43} \mathrm{On}$ the other hand, DPAP emission is strongly solvatochromic, showing a red-shift of up to $120 \mathrm{~nm}$ when increasing the solvent polarity from cyclohexane $\left(\lambda_{\mathrm{em}}=430 \mathrm{~nm}\right)$, to $o$-xylene $\left(\lambda_{\mathrm{em}}=470 \mathrm{~nm}\right)$, to THF $\left(\lambda_{\mathrm{em}}=505 \mathrm{~nm}\right)$, and to acetonitrile $\left(\lambda_{\mathrm{em}}=550 \mathrm{~nm}\right)$. Beside such exceptional sensitivity toward solvent polarity, DPAP displays a noteworthy response to viscosity. ${ }^{43}$ In this respect, hampering DPAP intramolecular rotation upon increasing the viscosity of the medium prompts an increase of the rotor fluorescence intensity.

\section{Spectroscopic characterization of DPAP/polymer films}

DPAP was dispersed at different concentrations $(0.05-0.1 \mathrm{wt} . \%)$ in poly(methyl methacrylate) (PMMA) and polycarbonate (PC) films 
by film casting. These concentrations were selected to realize polymer films with emission intensities that are not impacted by spurious effects stemming from high rotor concentration such as aggregation, self-quenching, self-absorption, etc. ${ }^{52}$ The DPAP/polymer films have a thickness of 90-120 $\mu \mathrm{m}$, appear homogeneous, and have absorption features similar to those previously reported for DPAP in organic solvents (Fig. S1).

PMMA and PC are amorphous polymers with glass transition temperatures of about $100-110$ and $150{ }^{\circ} \mathrm{C}$, respectively. Moreover, they only gave rise to residual emission upon $325 \mathrm{~nm}$ photoexcitation. DPAP was dispersed in PMMA and PC glassy matrices, in which the rotor intramolecular rotation is strongly hampered. Under such conditions, the radiative decay of DPAP is expected to dominate its photophysics. Recently, Iasilli et al. documented that when tetraphenylethylene (TPE) is dispersed in a glassy polystyrene (PS) matrix, the reduced intramolecular rotation result in strong emission of the dye, whereas the emission is significantly weakened when viscous but not glassy polymer matrices are used. ${ }^{32}$ In line with these assumptions, both DPAP/polymer films gave rise to a similar bright blue-green emission characterized by a single unstructured broad band $(390-570 \mathrm{~nm})$ with maxima at about $450 \mathrm{~nm}$ (Fig. 2).

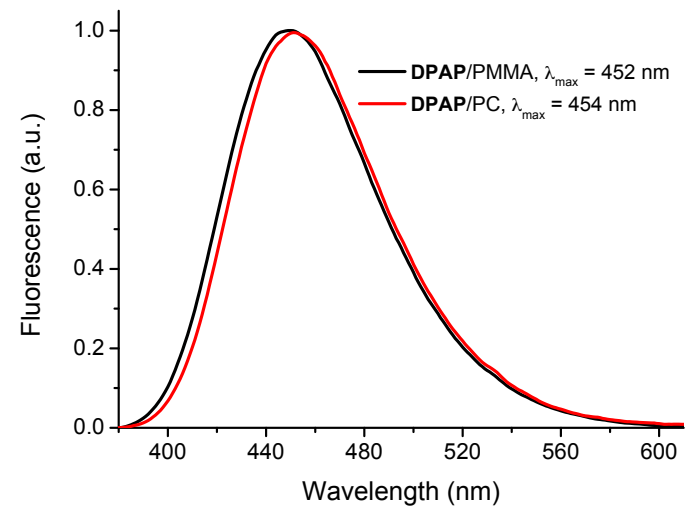

Fig. 2 Fluorescence emission spectra of 0.05 wt.\% DPAP/PMMA and DPAP/PC films $\left(\lambda_{\text {exc }}=325 \mathrm{~nm}\right)$.

Emission spectra of both films are almost identical with only a 2 $\mathrm{nm}$ difference in their maxima probably due to the similar dielectric constant of PMMA and PC (2.8 and 2.9, respectively). Likewise, the emission lifetimes of these DPAP/polymer films determined upon excitation at 297 or $403 \mathrm{~nm}$ were also similar, with values of 12.7 (PMMA) and $12.1 \mathrm{~ns}$ (PC) (Fig. S2). Note that the fluorescence lifetimes compare well with those obtained in lowpolar DPAP solutions in solvents such as $\mathrm{CH}_{2} \mathrm{Cl}_{2}$ or $o$-xylene. ${ }^{43}$

\section{Effects of VOC exposure on the optical emission of DPAP/PMMA} films

The DPAP/PMMA films were exposed to solvents with different vapour pressure, polarity index and Flory-Huggins interaction parameter $\chi$ (Table 1$)$. It is worth noting that $\chi$ is small in the case of effective solvent/polymer interactions. Owing to the fact that the solvent uptake is likely to affect the polymer matrix viscosity and polarity, a vapochromic effect was expected to play an appreciable role.

The emission spectra of DPAP/PMMA films exposed to $n$-hexane, that is, the least polar and interacting solvent with PMMA, are reported in Fig. S3. In particular, no changes in the film emission neither in intensity nor in position - was noted even after $38 \mathrm{~min}$. exposure to solvent vapours. ${ }^{53}$ In stark contrast to the aforementioned case, the DPAP/PMMA emission was strongly impacted upon exposure to more polar and PMMA-interacting VOCs. The progressive change in the emission of DPAP/PMMA films exposed to toluene and THF vapours is shown in Fig. 3.
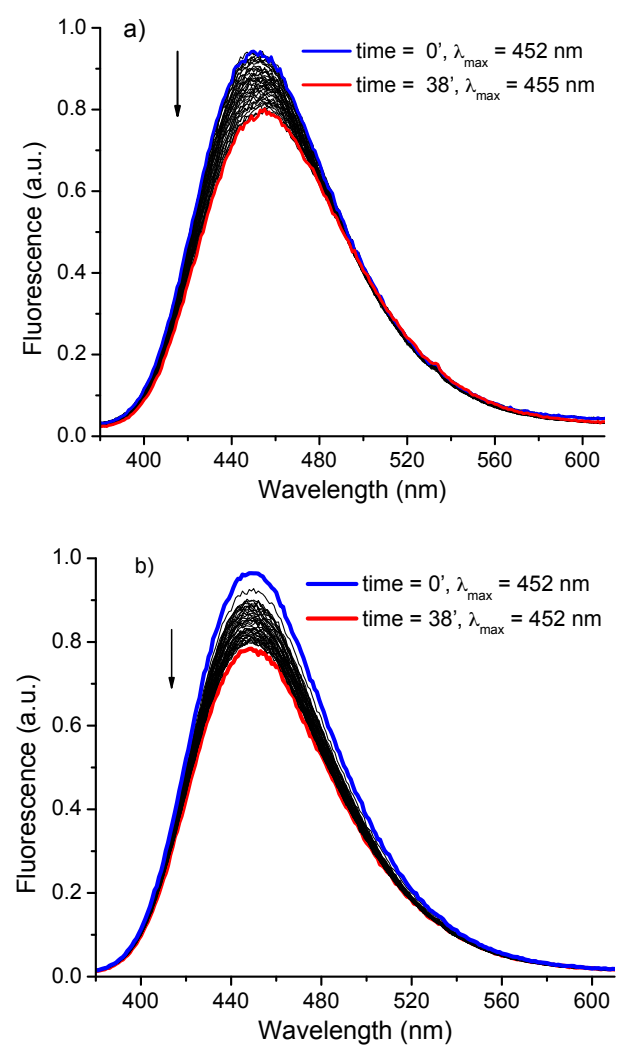

Fig. 3 Progressive changes in the fluorescence emission of 0.05 wt. \% DPAP/PMMA films as a function of exposure to (a) toluene and (b) THF vapours $\left(\lambda_{\text {exc }}=325 \mathrm{~nm}\right)$. The spectra were collected for $38 \mathrm{~min}$. with a time interval of $1 \mathrm{~min}$.

In both cases, the decrease in the DPAP/PMMA emission is mainly ascribed to the viscosity sensitivity of DPAP with increasing solvent uptake by the polymer matrix. Note that in the glassy state the PMMA matrix is characterized by a large fraction of free volume. The latter comes in the form of channels and cavities reaching molecular dimensions. Considering that solvent vapour fills these empty spaces, diffusion and swelling of the polymer starts from the outer surface layers inwards. In turn, an overall decrease of the local microviscosity evolves. ${ }^{54}$ This phenomenon leads to a partial increase of DPAP mobility, which, in turn, favours its nonradiative deactivation.

A more evident change in the emission is observed for DPAP/PMMA films exposed to either $\mathrm{CH}_{3} \mathrm{CN}$ or $\mathrm{CHCl}_{3}$ vapours (Fig. 4), solvents which present a favourable combination of polarity index and $\chi$ parameter (Table 1 ). When vapours of $\mathrm{CH}_{3} \mathrm{CN}$ and $\mathrm{CHCl}_{3}$ penetrate into the polymer films, their emission intensity dropped reaching a minimum after $5 \mathrm{~min}$. of vapour exposure. From there on, the emission remained unchanged. ${ }^{55} \mathrm{On}$ the other hand, for $\mathrm{CH}_{3} \mathrm{CN}$ and $\mathrm{CHCl}_{3}$ a red-shifted emission is observed (28 and $49 \mathrm{~nm}$, respectively). Interestingly, despite having the higher polarity index, $\mathrm{CH}_{3} \mathrm{CN}$ displays a lower red-shift than $\mathrm{CHCl}_{3}$. This finding can be rationalized considering that the magnitude of the red-shift in the DPAP-doped films emission upon solvent vapour exposure is the result of a subtle interplay between solvent polarity (vide supra) (3) $^{43}$ and solvent/polymer interactions.

The solvent permeation into the film appears crucial for establishing the response time of the film. Similar findings have been recently reported for PMMA films doped with solvatochromic dyes. The vapochromic response resulted substantially delayed when methanol, the weakest interacting solvent with the matrix, or its mixture with dichloromethane, was utilised. ${ }^{50}$

By contrast, vapour pressure appears unable to play an effective role in the phenomenon selectivity. 

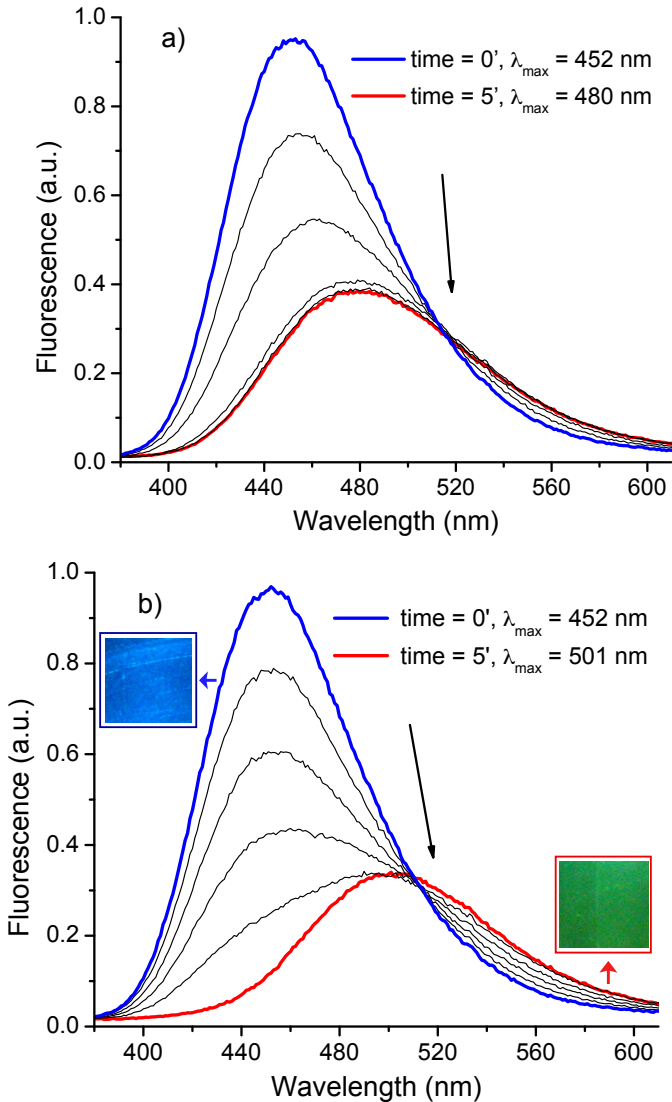

Fig. 4 Progressive changes in the fluorescence emission of 0.05 wt.\% DPAP/PMMA films as a function of exposure to (a) $\mathrm{CH}_{3} \mathrm{CN}$ and (b) $\mathrm{CHCl}_{3}\left(\lambda_{\text {exc }}=325 \mathrm{~nm}\right)$ and (inset) pictures of the same film with the exposure time of $\mathrm{CHCl}_{3}$ vapours taken under the illumination at $366 \mathrm{~nm}$. The spectra were collected for $5 \mathrm{~min}$. with a time interval of $1 \mathrm{~min}$.

In Fig. 5, the relative emission variations of DPAP/PMMA films as a function of solvent vapours exposure time indicate that the largest and fastest vapochromic response occurs for films that are exposed to VOCs featuring high polarity and strong chemical interactions with the polymer matrix such as $\mathrm{CHCl}_{3}$ and $\mathrm{CH}_{3} \mathrm{CN}$.

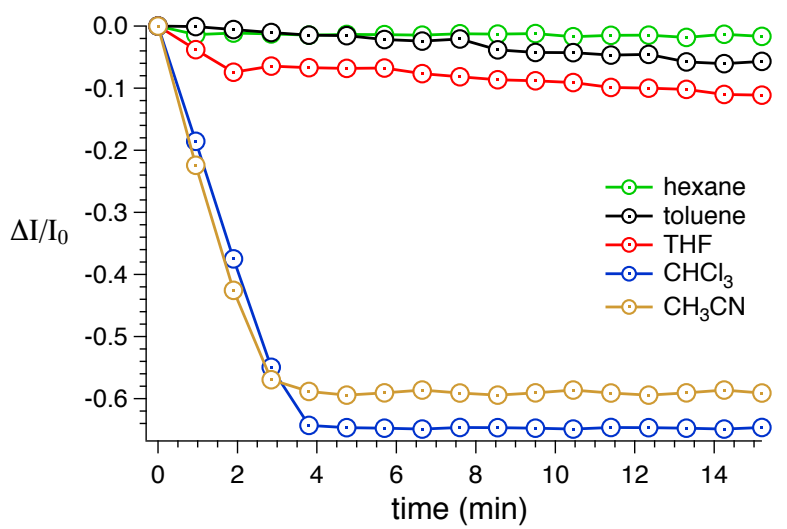

Fig. 5 Relative fluorescence intensity variation of peak maximum $\left(\Delta \mathrm{I} / \mathrm{I}_{0}\right)$ of $0.05 \mathrm{wt} . \%$ DPAP/PMMA films as a function of exposure time to different VOCs $\left(\lambda_{\mathrm{exc}}=325 \mathrm{~nm}\right)$.

These results suggest that the selectivity of DPAP/PMMA films is determined by the chemical affinity of PMMA for the solvent vapours and by the solvent polarity. More specifically, solvents with $\chi$ values lower than 0.45 and polarity indices higher than 4 interact well with the PMMA matrix, thus providing the vapochromic behaviour.
The kinetics of the vapochromic phenomenon was also affected by the DPAP concentration within the doped films. When $0.1 \mathrm{wt} . \%$ DPAP/PMMA films were exposed to $\mathrm{CHCl}_{3}$, the minimum emission intensity was reached after a $16 \mathrm{~min}$. exposure (Fig. S5), as compared to $5 \mathrm{~min}$. at lower rotor concentration.

Fig. 6 documents that the vapochromism of DPAP/PMMA films is completely reversible. After desorption of $\mathrm{CHCl}_{3}$ from the DPAP/PMMA films, the original emission is reinstalled and a second cycle of solvent exposure resulted in the same change in emission as observed during the first cycle. A similar reversible behaviour was observed for $\mathrm{CH}_{3} \mathrm{CN}$ (data not shown).

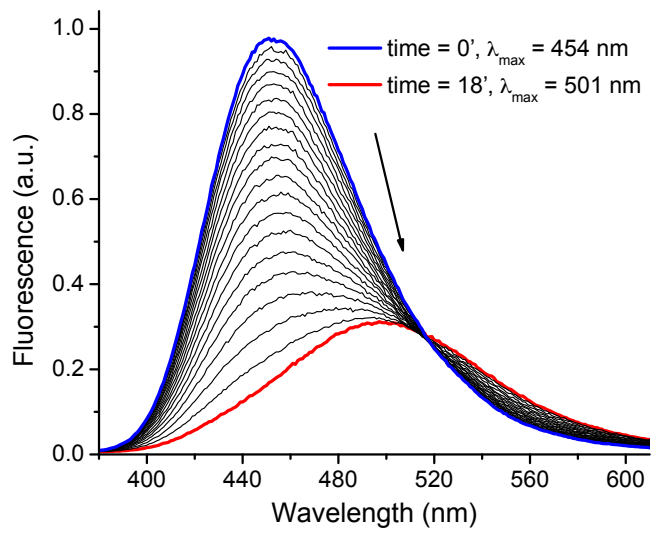

Fig. 6 Progressive changes in the emission of 0.1 wt.\% DPAP/PMMA films as a function of exposure to a second cycle of $\mathrm{CHCl}_{3}$ vapours $\left(\lambda_{\text {exc }}=325 \mathrm{~nm}\right)$. The spectra were collected for 18 min. with a time interval of $1 \mathrm{~min}$.

Effects of VOC exposure on the optical emission of DPAP/PC films

Considering that the Flory-Huggins interaction parameter $\chi$ is unavailable for some solvent/PC combinations, we used a semiempirical relationship to predict solvent/PC interactions, which takes into account the solubility parameter difference $\Delta \delta$, that is, the measure of the attractive strength between molecules of the material. ${ }^{48}$ Notably, the $\Delta \delta\left(\Delta \delta=\delta_{\mathrm{PC}}-\delta_{\text {solvent }}\right)$ is small for effective solvent/PC interactions (Table 1).

In Fig. S7, the emission spectra of DPAP/PC films upon exposure to $n$-hexane as a function of time are presented. Similar to DPAP/PMMA, DPAP/PC films revealed no appreciable alterations in terms of their emission maximum and intensity even after $38 \mathrm{~min}$. of solvent exposure.

When $\mathrm{CH}_{3} \mathrm{CN}$ is used, the emission of the DPAP/PC films experiences a significant quenching and red-shift $(25 \mathrm{~nm})$, similar to the DPAP/PMMA (Fig. 7).

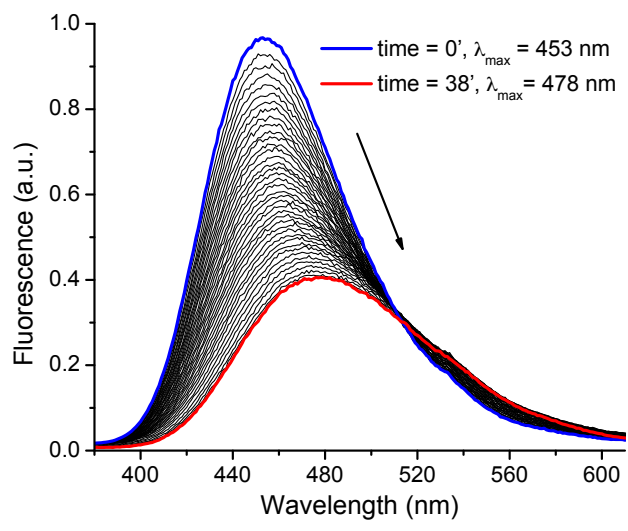

Fig. 7 Progressive changes in the emission of 0.05 wt. $\%$ DPAP/PC films as a function of exposure to $\mathrm{CH}_{3} \mathrm{CN}$ vapours $\left(\lambda_{\mathrm{exc}}=325 \mathrm{~nm}\right)$. 
The spectra were collected for 38 min. with a time interval of 55 seconds.

An even more intriguing vapochromic response was noticed when highly PC-interacting solvents such as toluene, THF, and $\mathrm{CHCl}_{3}$ were used (Fig. 8).
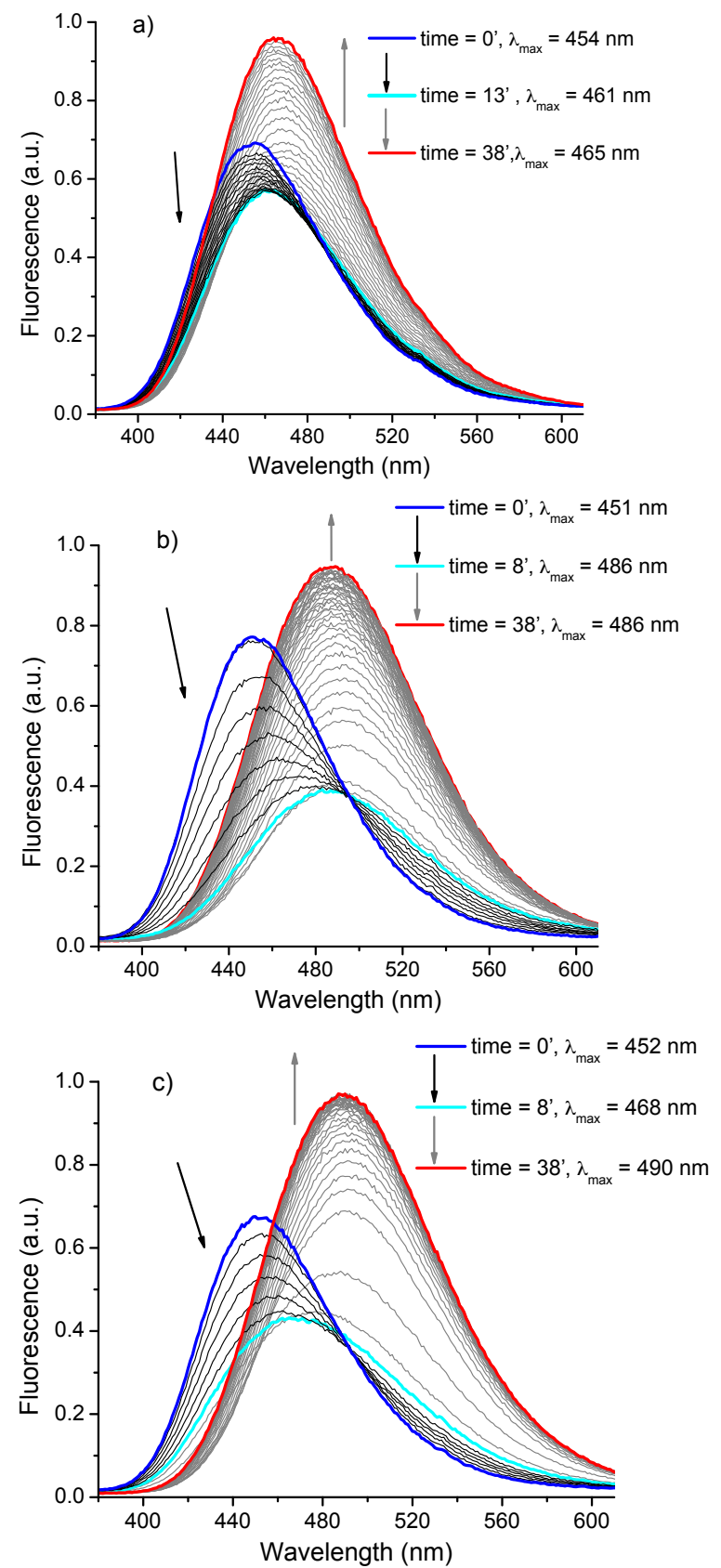

Fig. 8 Progressive changes in the emission of $0.05 \mathrm{wt} . \%$ DPAP/PC films as a function of exposure to (a) toluene, (b) THF, and (c) $\mathrm{CHCl}_{3}$ vapours $\left(\lambda_{\text {exc }}=325 \mathrm{~nm}\right.$ The spectra were collected for 38 min. with a time interval of $1 \mathrm{~min}$.

Toluene, THF, and $\mathrm{CHCl}_{3}$ are an inception to a rather unexpected emission behaviour. Following an initial drop in DPAP/PC emission intensity at short solvent vapours exposure times, a marked fluorescence enhancement combined with a red-shift was observed. To this end, emission intensities recorded after $38 \mathrm{~min}$. revealed an increase going from toluene to THF and $\mathrm{CHCl}_{3}$. This trend is in accordance with the solubility parameter differences of these solvents.
Notably, the aforementioned emission enhancement occurred already after 7-8 min. of $\mathrm{CHCl}_{3}$ exposure, whereas it appeared slower for THF (9-10 min.) and toluene (13-14 min.) (Fig. 9).

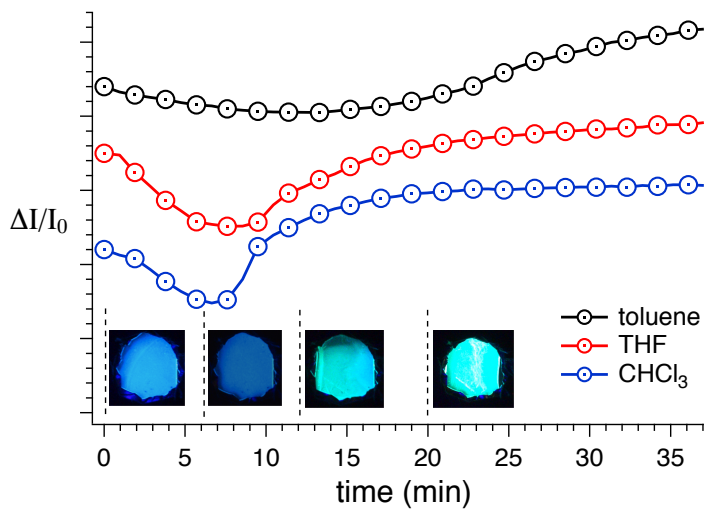

Fig. 9 Relative fluorescence intensity variation of peak maximum $\left(\Delta \mathrm{I} / \mathrm{I}_{0}\right)$ of the $0.05 \mathrm{wt} . \% \mathrm{DPAP} / \mathrm{PC}$ films as a function of the exposure time to toluene, THF and $\mathrm{CHCl}_{3}$ vapors $\left(\lambda_{\text {exc }}=325 \mathrm{~nm}\right)$. Pictures of the same DPAP/PC film at $0,6,12$ and $20 \mathrm{~min}$. (from left to right) of exposure time to $\mathrm{CHCl}_{3}$ vapors. The pictures were taken under the illumination at $366 \mathrm{~nm}$.

Interestingly, the emission red-shift maximizes for DPAP/PC films exposed to $\mathrm{CHCl}_{3}$ vapours $(38 \mathrm{~nm})$ which, compared to toluene and THF, is the solvent with the higher polarity index and lower PC-solubility parameter difference $\Delta \delta$. Such changes in the DPAP/PC film emission could be visualized by naked-eye (Fig. 9). On the other hand, the smaller emission red-shift $(11 \mathrm{~nm})$ was observed for films exposed to toluene, which has the lower polarity index and the higher $\Delta \delta$ value.

Inspection of the DPAP/PC films immediately after $\mathrm{CHCl}_{3}$ exposure under visible light revealed an evident change of film morphology (Fig. 10a). In the area exposed to $\mathrm{CHCl}_{3}$, the films gave rise to a clear whitening combined with an evident embrittlement. These morphological changes are accompanied by a strong modification of the emitted light of the film from blue to intense green. Next, emission imaging studies were carried out investigating the morphology of DPAP/PC films before (Fig. 10b) and after (Fig. 10c) $\mathrm{CHCl}_{3}$ exposure. In particular, the films were subjected to solvent vapours for $38 \mathrm{~min}$. and analysed by means of confocal scanning laser fluorescence microscopy using a laser source at 405 $\mathrm{nm}$ and an emission in the $430-490 \mathrm{~nm}$ range.
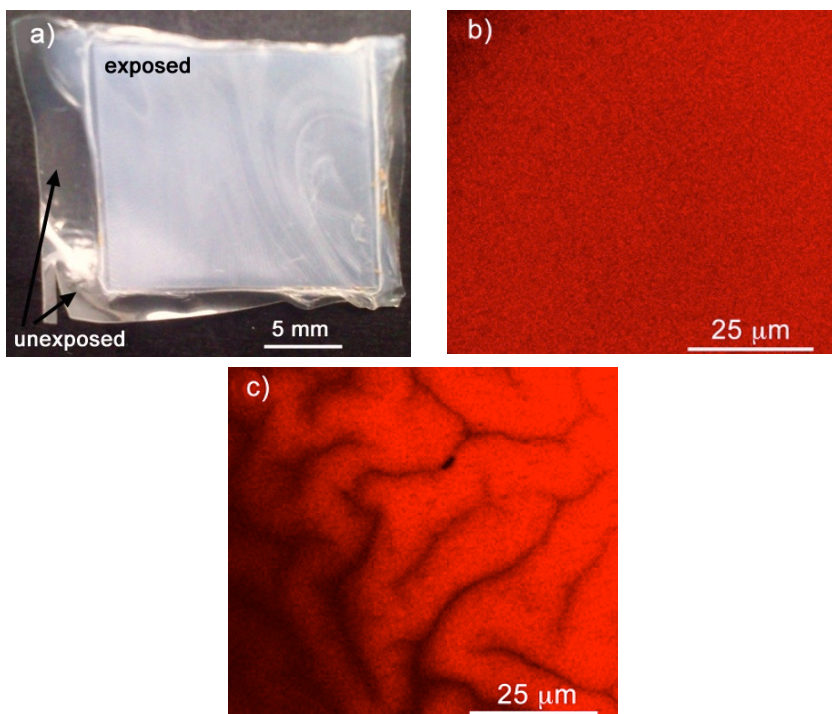
Fig. 10 (a) Digital picture of 0.05 wt.\% DPAP/PC film immediately after exposure to $\mathrm{CHCl}_{3}$ vapours for $38 \mathrm{~min}$. under visible light. Confocal microscope images of DPAP/PC films with emission collection in the 430-490 $\mathrm{nm}$ range (b) before and (c) after exposure to $\mathrm{CHCl}_{3}$ vapours for $38 \mathrm{~min}$. In this latter case, after the $\mathrm{CHCl}_{3}$ vapour exposure, the DPAP/PC film was placed at room temperature and atmospheric pressure for $40 \mathrm{~min}$. allowing the desorption of the trapped solvent molecules from the polymeric matrix. Note that the emission images are in pseudocolors.

Prior to solvent exposure, the film surface appears smooth and homogeneous. After solvent exposure, the area of the film placed in contact with $\mathrm{CHCl}_{3}$ revealed a strong modification of its texture, the appearance of numerous surface cracks, and a significant increase in the emission.

The effect of the solvent desorption on the emission properties of the $\mathrm{CHCl}_{3}$-exposed DPAP/PC films was also investigated. In this case, the original DPAP/PC emission maximum was almost completely recovered upon solvent desorption $(458 \mathrm{~nm}$ (Fig. 11a, red spectrum) versus $452 \mathrm{~nm}$ (Fig. 11a, green spectrum) for $\mathrm{CHCl}_{3}-$ exposed and pristine DPAP/PC films, respectively). In other words, when $\mathrm{CHCl}_{3}$ starts to desorb from the doped matrix, the film emission is accordingly shifted to lower wavelengths in line with the different effective polarity of $\mathrm{PC}$ and $\mathrm{CHCl}_{3}$. However, to our surprise, after $\mathrm{CHCl}_{3}$ desorption DPAP/PC films showed a fluorescence intensity that was much higher than that of the pristine film before solvent exposure (Fig. 11a, red spectrum).

The second cycle of $\mathrm{CHCl}_{3}$ exposure showed a similar trend to the first cycle, that is, a film fluorescence emission drop flanked by a red-shift of the emission maximum, but without the marked fluorescence enhancement (Fig. 11b). Similar results were also registered when using toluene (data not shown) and THF (Fig. S8-S10), highly interacting VOCs for PC.
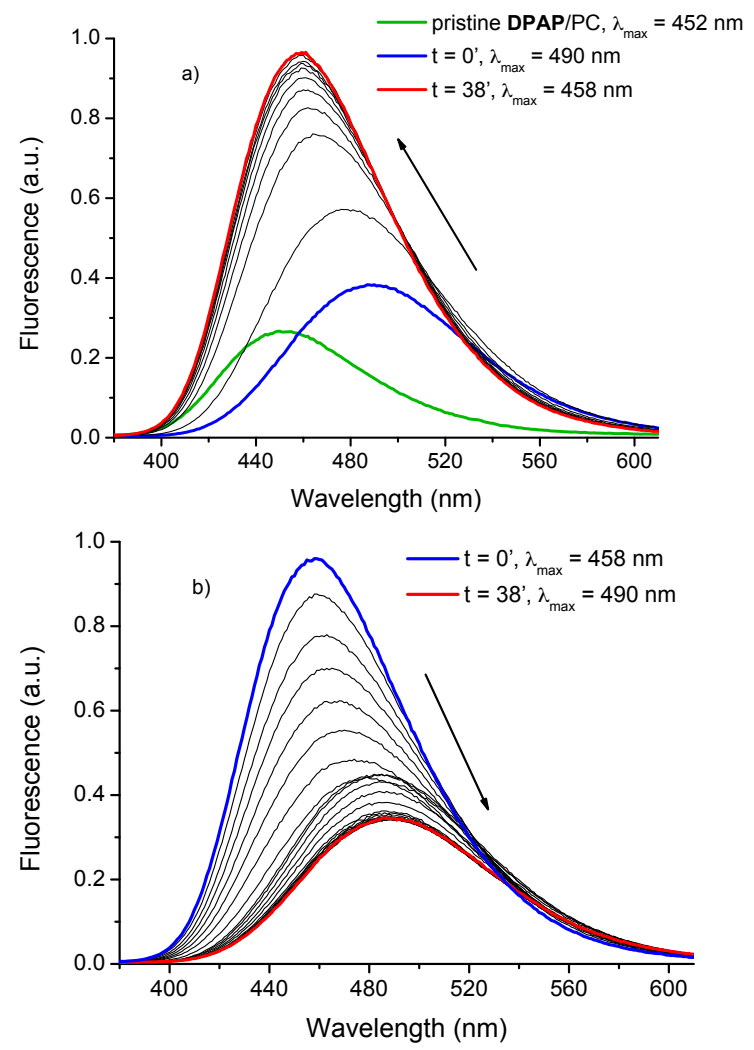

Fig. 11 Progressive changes in the emission of $\mathrm{CHCl}_{3}$-exposed 0.05 wt.\% DPAP/PC films (after equilibration in the presence of the same solvent vapors) as a function of (a) $\mathrm{CHCl}_{3}$ desorption of a $\mathrm{CHCl}_{3}$-exposed DPAP/PC film after equilibration in the presence of the same solvent vapors. The emission spectrum of the pristine DPAP/PC film is shown in green. (b) Second cycle exposure to
$\mathrm{CHCl}_{3}$ vapors $\left(\lambda_{\text {exc }}=325 \mathrm{~nm}\right)$. In both experiments the spectra were collected for $38 \mathrm{~min}$. with a time interval of $1 \mathrm{~min}$.

We hypothesize that the phenomenon associated with the emission increase of the DPAP/PC films noted during the first $\mathrm{CHCl}_{3}$ exposure cycle could be related to changes in the internal flexibility of DPAP triggered by some irreversible solvent-induced modification of the PC matrix at the molecular level. More specifically, an increase of the viscosity of the solvent-exposed PC matrix is expected to occur which could lead to an increase in the rotor fluorescence intensity. Changes with respect to the appearances of DPAP/PC films upon solvent exposure, that is, from transparent to opaque, suggest crystallization of the polymeric matrix. Crystallization, in turn, is likely to hamper the internal motion of DPAP present in the PC phase.

To validate our hypothesis, we probed the thermal properties of DPAP/PC films before and after $\mathrm{CHCl}_{3}$ exposure by comparing their first heating DSC scans (Fig. 12).

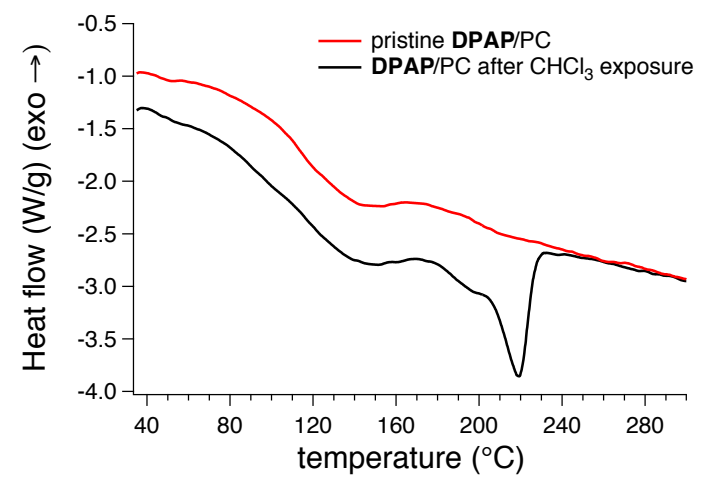

Fig. 12 First heating DSC scans of pristine (red trace) and $\mathrm{CHCl}_{3}$ treated (black trace) $0.05 \mathrm{wt} \%$ DPAP/PC films. The $\mathrm{CHCl}_{3-}$ treated films were prepared exposing the DPAP/PC films to $\mathrm{CHCl}_{3}$ vapours for $40 \mathrm{~min}$. and the resulting films let to stand at room temperature and atmospheric pressure for $40 \mathrm{~min}$. in order to eliminate solvent molecules trapped in the polymeric matrix.

During the first heating scan both pristine and $\mathrm{CHCl}_{3}$-exposed films displayed a glass transition temperature $\left(\mathrm{T}_{\mathrm{g}}\right)$ at around 150 ${ }^{\circ} \mathrm{C}$. However, in contrast to the pristine DPAP/PC films which may be regarded essentially as amorphous materials, the solventexposed system showed a strong endothermic peak at around 220 ${ }^{\circ} \mathrm{C}$ which supports the existence of a crystalline phase.

The crystallization of PC is of considerable interest and has been investigated in the literature. ${ }^{51} \mathrm{PC}$ features poor crystallization because of its rigid macromolecular chains. Attempts have been made to induce crystallization of PC by exposure to organic solvents in the vapour or liquid state. ${ }^{56-58}$ Under these conditions, the crystallization rate was strongly enhanced due to the role of the absorbed solvent as plasticizers. As such, their presence increases the mobility of polymer segments and, thus, enables crystallization to occur even at room temperature. The crystallinity $\left(\mathrm{f}_{\mathrm{c}}\right)$ of PC, as a function of $\mathrm{CHCl}_{3}$ or THF exposure was calculated using Eq. 1 (see experimental part) and is reported in Table 2.

Table 20.05 wt.\% DPAP/PC crystallinity $\left(f_{c}\right)$ as a function of $\mathrm{CHCl}_{3}$ and THF vapor exposure time.

\begin{tabular}{|c|c|c|c|}
\hline Sample & solvent & time $(\min )$ & $f_{\mathrm{c}}(\%)$ \\
\hline DPAP/PC & - & - & 0 \\
\hline DPAP/PC & $\mathrm{CH}_{3} \mathrm{CN}$ & 30 & $\sim 0$ \\
\hline DPAP/PC & THF & 30 & 7.15 \\
\hline DPAP/PC & $\mathrm{CHCl}_{3}$ & 10 & 2.00 \\
\hline DPAP/PC & $\mathrm{CHCl}_{3}$ & 30 & 9.38 \\
\hline
\end{tabular}


After $10 \mathrm{~min}$. of exposure to $\mathrm{CHCl}_{3}$, DPAP/PC films exhibited only $2 \%$ of crystallinity, which increased and plateaued at $9 \%$ with increasing the vapour exposure time up to $30 \mathrm{~min}$. This suggests that small changes in the PC crystallinity do not affect significantly the viscosity of the doped Pc matrix as documented by the decrease in the rotor emission intensity in the film, which is mainly related to the polarity of the environment. On the other hand, at longer vapour exposure a higher degree of crystallinity is reached, which in turn induces an increased viscosity in the matrix and an enhancement in DPAP emission intensity. Additionally, the impact of THF on both the emission and crystallinity of the rotor-doped films was smaller than with $\mathrm{CHCl}_{3}$, which is in agreement with its higher solubility parameter difference $\Delta \delta$ (Table 1 ). Prolonged exposure to VOCs for longer than $38 \mathrm{~min}$. did not cause detectable variations of both the emission response and phase behaviour of the PC matrix.

Compared to PMMA, the selectivity of DPAP/PC films is more determined by the chemical affinity of $\mathrm{PC}$ for the solvent vapours since acetonitrile, the most polar solvent, is not able to induce polymer crystallization.

Interestingly, DPAP emission studies in mixtures of $o$-xylene and silicone oil 500, whose polarity is comparable with that of PC, corroborate the findings made with DPAP/PC films. With increasing solvent viscosity, that is, increasing the content of silicone oil in the mixtures, a similar emission enhancement evolved (Fig. S11). Changing the viscosity resulted, however, in no appreciable changes of the $12.5 \mathrm{~ns}$ emission lifetimes (Fig. S12). Both trends are in good agreement with the results gathered for the DPAP/PC films. Note that DPAP/PC films before and after THF exposure reveal the same lifetimes of around $12.1 \mathrm{~ns}$ (Fig. S10). ${ }^{59}$ This substantiates the hypothesis of a hindered mobility of DPAP when going from amorphous to crystalline PC.

\section{Conclusions}

We have demonstrated that a FMR, namely DPAP, characterized by high sensitivity toward solvent polarity and viscosity, once embedded into plastic materials, confers new vapochromic characteristics to the resulting films.

DPAP exhibits viscosity- and polarity-dependent emission properties when dispersed at low loadings $(<0.1 \mathrm{wt} . \%)$ in PMMA plastic films and exposed to saturated atmospheres of polar and wellinteracting VOCs. These films showed a significant decrease and red-shift of their emission due to solvent-induced changes in the local polarity and viscosity of the medium. On the contrary, the film emission remained unaffected when low polar and barely interacting VOCs were tested. The overall vapochromic response of these DPAP/polymer films was even more marked when PC was used. In this case, an initial solvent-induced red-shift and decrease in emission, as observed for the PMMA-based systems, was accompanied by a further red-shift and increase in the film fluorescence intensity at longer solvent-exposure time. This effect is attributed to a polymer crystallization process in the plastic films upon solvent uptake, which provokes a general increase in the viscosity of the matrix. This process reduces the flexibility of the embedded DPAP, hampering its internal motions and, in turn, enhancing its radiative decay process.

In light of this peculiar response, DPAP-enriched plastic films respond to vapours of different organic solvents, providing a reproducible emission signal even after several successive cycles of vapour exposure. Solvent permeation into the film is crucial in establishing the response time of the film.

These findings consistently support the effective preparation and use of a new class of vapochromic plastic materials. Further work will be focused on the validation of the proposed approach towards a more extended set of solvents and physical conditions. Also, this aims at the determination of the sensitivity and the detection limit of the dye/polymer films.

\section{Acknowledgements}

The research leading to these results has received funding from the European Union's Seventh Framework Programme (FP7/20072013) under grant agreement No. ERC-2012-AdG-320951DREAMS. This work was partially supported by MIUR through FIRB program Futuro in Ricerca "SUPRACARBON" (contract $\mathrm{n}^{\circ}$ RBFR10DAK6) and by the Spanish MICINN (CTQ201124187/BQU). A.P. acknowledges SABIC for the SABIC Innovation Challenge grant within the area of smart polymer materials.

\section{Notes and references}

${ }^{a}$ Scuola Normale Superiore, Piazza dei Cavalieri 7, I-56126 Pisa, Italy ${ }^{b}$ Department of Chemistry and Pharmacy \& Interdisciplinary Center for Molecular Materials (ICMM), Friedrich-Alexander-Universität ErlangenNürnberg, 91058, Erlangen, Germany

${ }^{\mathrm{c}}$ NEST, Scuola Normale Superiore and Istituto Nanoscienze-CNR, Pisa, Italy

${ }^{\mathrm{d} D e p a r t a m e n t o ~ d e ~ Q u i ́ m i c a ~ O r g a ́ n i c a, ~ U n i v e r s i d a d ~ A u t o ́ n o m a ~ d e ~ M a d r i d, ~}$ 28049, Cantoblanco, Spain; e-mail: giovanni.bottari@uam.es

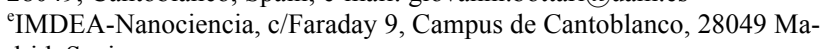
drid, Spain

${ }^{\mathrm{f}}$ Dipartimento di Chimica e Chimica Industriale, Università di Pisa, Pisa, Italy; e-mail: andrea.pucci@unipi.it

${ }^{\mathrm{g}}$ INSTM, UdR Pisa, Italy

Electronic Supplementary Information (ESI) available: additional fluorescence experiments on DPAP/polymer films as well as life-time curves. See DOI: $10.1039 / \mathrm{b} 000000 \mathrm{x} /$

1. J. L. Adgate, B. D. Goldstein and L. M. McKenzie, Environ. Sci. Technol., 2014, 48, 8307-8320.

2. Y. M. Kim, S. Harrad and R. M. Harrison, Environ. Sci. Technol., 2001, 35, 997-1004.

3. S. Endo, B. I. Escher and K.-U. Goss, Environ. Sci. Technol., 2011, 45, 5912-5921.

4. S. Manzetti, E. R. van der Spoel and D. van der Spoel, Chem. Res. Toxicol., 2014, 27, 713-737.

5. C. Elosua, C. Bariain, A. Luquin, M. Laguna and I. R. Matias, Sens. Actuators, B, 2011, 157, 388-394.

6. C. Elosua, C. Bariain, I. R. Matias, F. J. Arregui, E. Vergara and M. Laguna, Sens. Actuators, B, 2009, 137, 139-146.

7. A. K. Srivastava, Sens. Actuators, B, 2003, 96, 24-37.

8. N. A. Rakow and K. S. Suslick, Nature, 2000, 406, 710-713.

9. M. C. Janzen, J. B. Ponder, D. P. Bailey, C. K. Ingison and K. S. Suslick, Anal. Chem., 2006, 78, 3591-3600.

10. D. T. McQuade, A. E. Pullen and T. M. Swager, Chem. Rev., 2000, 100, 2537-2574.

11. S. W. Thomas, G. D. Joly and T. M. Swager, Chem. Rev., 2007, 107, 1339-1386.

12. J. Yoon, S. K. Chae and J.-M. Kim, J. Am. Chem. Soc., 2007, 129, 3038-3039.

13. A. Pucci, G. Ruggeri, S. Bronco, M. Bertoldo, C. Cappelli and F. Ciardelli, Progr. Org. Coat., 2007, 58, 105-116.

14. A. Pucci, N. Tirelli, G. Ruggeri and F. Ciardelli, Macromol. Chem. Phys., 2005, 206, 102-111.

15. A. Pucci, T. Biver, G. Ruggeri, L. Itzel Meza and Y. Pang, Polymer, 2005, 46, 11198-11205.

16. F. Ciardelli, G. Ruggeri and A. Pucci, Chem. Soc. Rev., 2013, 42, 857-870.

17. A. Pucci, R. Bizzarri and G. Ruggeri, Soft Matter, 2011, 7, 3689-3700.

18. A. Pucci and G. Ruggeri, J. Mater. Chem., 2011, 21, 82828291.

19. J. R. Kumpfer, S. D. Taylor, W. B. Connick and S. J. Rowan, J. Mater. Chem., 2012, 22, 14196-14204.

20. S. H. Lim, L. Feng, J. W. Kemling, C. J. Musto and K. S. Suslick, Nat. Chem., 2009, 1, 562-567.

21. C.-M. Che, W.-F. Fu, S.-W. Lai, Y.-J. Hou and Y.-L. Liu, Chem. Commun., 2003, 118-119.

22. M. A. H. Alamiry, E. Bahaidarah, A. Harriman, T. Bura and R. Ziessel, RSC Adv., 2012, 2, 9851-9859.

23. M. A. Haidekker, W. Akers, D. Lichlyter, T. P. Brady and E. A. Theodorakis, Sensor Lett, 2005, 3, 42-48.

24. M. A. Haidekker, T. P. Brady, D. Lichlyter and E. A. Theodorakis, Bioorg. Chem., 2005, 33, 415-425. 
25. A. Mustafic, H.-M. Huang, E. A. Theodorakis and M. A. Haidekker, J. Fluoresc., 2010, 20, 1087-1098.

26. M. A. Haidekker and E. A. Theodorakis, J. Biol. Eng., 2010, 4, No pp given.

27. M. A. Haidekker, M. Nipper, A. Mustafic, D. Lichlyter, M. Dakanali and E. A. Theodorakis, Springer Ser. Fluoresc., 2010, 8, 267308.

28. M. A. Haidekker and E. A. Theodorakis, Org. Biomol. Chem., 2007, 5, 1669-1678.

29. X. Zhang, B. Li, Z.-H. Chen and Z.-N. Chen, J. Mater. Chem., 2012, 22, 11427-11441.

30. Y. Hong, J. W. Y. Lam and B. Z. Tang, Chem. Commun., 2009, 4332-4353.

31. Y. Hong, J. W. Y. Lam and B. Z. Tang, Chem. Soc. Rev., 2011, 40, 5361-5388.

32. G. Iasilli, A. Battisti, F. Tantussi, F. Fuso, M. Allegrini, G. Ruggeri and A. Pucci, Macromol. Chem. Phys., 2014, 215, 499-506.

33. L. Liu, Y. Shao, J. Peng, C. Huang, H. Liu and L. Zhang, Anal. Chem., 2014, 86, 1622-1631.

34. A. Qin, J. W. Y. Lam and B. Z. Tang, Prog. Polym. Sci., 2012, 37, 182-209.

35. J. Wu, W. Liu, J. Ge, H. Zhang and P. Wang, Chem. Soc. Rev., 2011, 40, 3483-3495.

36. X. Zhang, M. Liu, B. Yang, X. Zhang and Y. Wei, Colloid Surf. B-Biointerfaces, 2013, 112, 81-86.

37. F. Zhou, J. Shao, Y. Yang, J. Zhao, H. Guo, X. Li, S. Ji and Z. Zhang, Eur. J. Org. Chem., 2011, 2011, 4773-4787, S4773/4771S4773/4770.

38. L.-L. Zhu, X. Li, F.-Y. Ji, X. Ma, Q.-C. Wang and H. Tian, Langmuir, 2009, 25, 3482-3486.

39. L.-L. Zhu, D.-H. Qu, D. Zhang, Z.-F. Chen, Q.-C. Wang and H. Tian, Tetrahedron, 2010, 66, 1254-1260.

40. X. Zhang, Z. Chi, H. Li, B. Xu, X. Li, S. Liu, Y. Zhang and J. Xu, J. Mater. Chem., 2011, 21, 1788-1796.

41. X. Zhang, Z. Chi, H. Li, B. Xu, X. Li, W. Zhou, S. Liu, Y. Zhang and J. Xu, Chem.-Asian J., 2011, 6, 808-811.

42. X. Zhang, Z. Chi, Y. Zhang, S. Liu and J. Xu, J. Mater. Chem. C, 2013, 1, 3376-3390.

43. M. Koenig, G. Bottari, G. Brancato, V. Barone, D. M. Guldi and T. Torres, Chem. Sci., 2013, 4, 2502-2511.

44. M. Koenig, T. Torres, V. Barone, G. Brancato, D. M. Guldi and G. Bottari, Chem. Commun., accepted manuscript.

45. D. R. Lide, Handbook of Chemistry and Physics, 88th edn., CRC Press, Boca Raton, FL, 2007-2008.

46. D. R. Lide, CRC Handbook of Chemistry and Physics, 86th Edition, CRC Press LLC, Boca Raton, FL, USA, 2004.

47. J. E. Mark, Physical Properties of Polymers Handbook, 2nd edn., Springer Science + Business Media, LLC, New York, 2007.

48. J. Brandrup, E. H. Immergut and E. A. Grulke, eds., Polymer Handbook, 4th edn., John Wiley \& Sons, Inc., New York, 1999.

49. V. P. Budtov, N. G. Bel'nikevich and L. S. Litvinova, Polym. Sci. Ser. A, 2010, 52, 362-367.

50. I. Platonova, A. Branchi, M. Lessi, G. Ruggeri, F. Bellina and A. Pucci, Dyes and Pigments, 2014, 110, 249-255.

51. Z. Fan, C. Shu, Y. Yu, V. Zaporojtchenko and F. Faupel, Polym. Eng. Sci., 2006, 46, 729-734.

52. DPAP concentrations lower than $0.05 \mathrm{wt} \%$, i.e. $0.02 \mathrm{wt} \%$, did not provide reliable results in terms of reproducibility of the luminescent response.

53. The slight decrease of emission intensity is not attributable to DPAP photodegradation, since no intensity change was recorded with multiple excitation scans over the investigated period in the absence of solvent vapors (Figures S4 (for DPAP/PMMA films) and S6 (for DPAP/PC films)).

54. B. Valeur and M. N. Berberan-Santos, Molecular Fluorescence: Principles and Applications, Wiley-VCH, Weinheim (Germany), 2012.

55. Solvent adsorption marginally affected film thickness, whose distance from the optical fiber remained unaltered. As a consequence, its contribution to fluorescence intensity change was neglected.

56. L. B. O. d. O. Flavia, C. A. M. L. Marcia, O. C. Lessandra and R. C. Thiago, Polym. Bull., 2011.
57. J. P. Mercier, G. Groeninckx and M. Lesne, J. Polym. Sci., Part C: Polym. Symp., 1967, 16, 2059-2067.

58. W. V. Titow, M. Braden, B. R. Currell and R. J. Loneragan, J. Appl. Polym. Sci., 1974, 18, 867-886.

59. Note that the emission lifetimes were gathered with dry DPAP/PC films, in which the adsorbed solvent was completely desorbed from the film. 


\section{Table of contents}

The reversible, vapochromic response of polymeric films doped with 4-(diphenylamino)phthalonitrile (DPAP) fluorescent molecular rotor suitable for sensing volatile organic compounds (VOCs) is presented

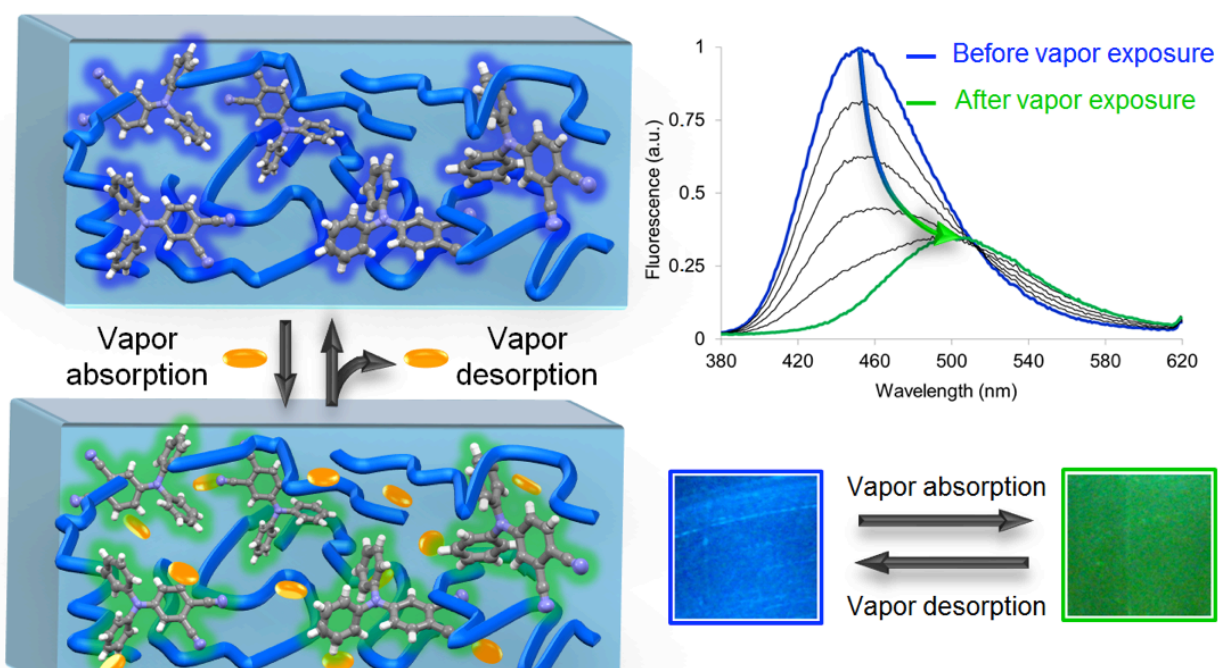

